\title{
Diastolic dysfunction in diabetes and the metabolic syndrome: promising potential for diagnosis and prognosis
}

\author{
H. von Bibra • M. St John Sutton
}

Received: 19 November 2009 / Accepted: 6 January 2010/Published online: 30 March 2010

(C) The Author(s) 2010. This article is published with open access at Springerlink.com

\begin{abstract}
Cardiac disease in diabetes mellitus and in the metabolic syndrome consists of both vascular and myocardial abnormalities. The latter are characterised predominantly by diastolic dysfunction, which has been difficult to evaluate in spite of its prevalence. While traditional Doppler echocardiographic parameters enable only semiquantitative assessment of diastolic function and cannot reliably distinguish perturbations in loading conditions from altered diastolic functions, new technologies enable detailed quantification of global and regional diastolic function. The most readily available technique for the quantification of subclinical diastolic dysfunction is tissue Doppler imaging, which has been integrated into routine contemporary clinical practice, whereas cine magnetic resonance imaging (CMR) remains a promising complementary research tool for investigating the molecular mechanisms of the disease. Diastolic function is reported to vary linearly with age in normal persons, decreasing by $0.16 \mathrm{~cm} / \mathrm{s}$ each year. Diastolic function in diabetes and the metabolic syndrome is determined by cardiovascular risk factors that alter myocardial stiffness and myocardial energy availability/bioenergetics. The latter is corroborated by the improvement in diastolic function with improvement in metabolic control of diabetes by specific medical therapy or lifestyle modification. Accordingly, diastolic dysfunction
\end{abstract}

H. von Bibra $(\bowtie)$

Clinic for Endocrinology, Diabetes and Vascular Medicine,

Klinikum Bogenhausen, Städt. Klinikum München $\mathrm{GmbH}$,

Englschalkingerstrasse 77,

81925 Munich, Germany

e-mail: von-Bibra@extern.lrz-muenchen.de

M. St John Sutton

Department of Medicine, Cardiovascular Division,

School of Medicine, University of Pennsylvania,

Philadelphia, PA, USA reflects the structural and metabolic milieu in the myocardium, and may allow targeted therapeutic interventions to modulate cardiac metabolism to prevent heart failure in insulin resistance and diabetes.

Keywords Cardiovascular disease - Diastolic dysfunction . Metabolic syndrome $\cdot$ Review . Tissue Doppler imaging · Type 2 diabetes
Abbreviations
BNP Brain natriuretic peptide
CAD Coronary artery disease
CMR Cine magnetic resonance imaging
$\mathrm{E}^{\prime} \quad$ Early diastolic myocardial velocity, derived from tissue Doppler imaging
E/E' Ratio of early diastolic velocities of mitral inflow (Doppler-derived) and myocardial movement (tissue Doppler imaging-derived) taken as left ventricular filling pressure
$\mathrm{S}^{\prime} \quad$ Systolic myocardial velocity, derived from tissue Doppler imaging

The metabolic syndrome and type 2 diabetes are associated with myocardial dysfunction

The metabolic syndrome is a cluster of risk factors of metabolic origin and has a growing prevalence worldwide $[1,2]$. The prototypical patient has non-specific symptoms, obesity and a sedentary lifestyle, and usually receives therapy for metabolic syndrome only after systemic hypertension and/or hyperlipidaemia is diagnosed. However, patients with the metabolic syndrome are at risk of multisystem disease and, accordingly, need early diagnosis 
and appropriate therapy before the full syndrome and its deleterious sequelae become established. Abdominal adiposity is commonly associated with insulin resistance and the development of a number of cardiovascular risk factors, prominent among which is diabetes mellitus [3, 4]. The most serious complication is coronary artery disease (CAD), which often occurs before overt diabetes mellitus is apparent [5] and when there is only subtle myocardial dysfunction that is manifest initially in diastole $[6,7]$. Because of accumulating evidence for predominantly diastolic dysfunction in diabetic patients without ischaemic, valvular and hypertensive disease, a specific diabetic cardiomyopathy has been proposed [8]. More recently, increased prevalence of diastolic dysfunction has also been shown in the metabolic syndrome [9].

Early subclinical diastolic dysfunction has only recently been recognised and its predictive value for diastolic heart failure appreciated [10-12], having previously been regarded as benign [13]. Diastolic dysfunction is the most prominent characteristic of diabetic cardiomyopathy $[7,14$, $15]$ and heart failure is the most common cause of death $(66 \%)$ in patients with type 2 diabetes after their first myocardial infarction [16]. The traditional echocardiographic and Doppler techniques have proved inconclusive for the diagnosis of diastolic dysfunction in diabetic cardiomyopathy and in the metabolic syndrome because there is no consensus regarding definitive diagnostic criteria. Furthermore, evidence-based medicine lacks specific therapy for diastolic dysfunction in general and for diabetic cardiomyopathy in particular, as the commonly recommended treatment options for systolic heart failure have not proved effective [13, 17].

Hence, this review seeks to present an interdisciplinary approach to diastolic dysfunction in diabetes and the metabolic syndrome, focusing on the associated problems, pathophysiology, diagnostic modalities, therapeutic implications and clinical applications.

\section{Problems}

Prevalence, relevance and prognosis of diastolic dysfunction There is indisputable evidence for the high prevalence of predominantly diastolic myocardial dysfunction in individuals aged $>65$ years. This prevalence is $16 \%$ in the general population and $35 \%$ in individuals with the metabolic syndrome $[9,18]$. However, it is $50 \%$ in individuals with prediabetes and overt type 2 diabetes, $60 \%$ in patients with CAD and $70 \%$ in patients with both CAD and diabetes [1922]. Diastolic myocardial dysfunction with a normal left ventricular ejection fraction is clinically important because it accounts for approximately $50 \%$ of all hospital admissions for acute heart failure [12]. For the individual patient, diastolic dysfunction and diastolic heart failure mean impaired quality of life induced by the deterioration in exercise capacity that limits activities of daily living [23]. Patients with the metabolic syndrome and those with diabetes often present with exertional dyspnoea and reduced exercise tolerance, which are due directly to diastolic myocardial dysfunction $[9$, 22, 24, 25].

Abnormalities of myocardial relaxation, i.e. grade 1 diastolic dysfunction, confer a twofold increase in all-cause and cardiac mortality $[10,11]$. This observation has increased awareness of diastolic heart failure with a normal ejection fraction $[12,13]$. However, despite the impressive impact of isolated diastolic dysfunction upon mortality, there remains a lack of evidence-based treatment regimes for diastolic dysfunction.

Problems with clinical assessment Diastolic dysfunction associated with the metabolic syndrome or diabetes is not always detected early. The delay in diagnosis is due partly to patient-related factors and partly to medical oversight. Patients are often ashamed of being overweight, have difficulty losing weight, and become resigned to their physical limitations in daily life and reticent about discussing this problem with their medical practitioner. Furthermore, most diabetologists do not routinely assess levels of physical activity or signs and symptoms of diastolic dysfunction, such as progressive exertional dyspnoea or exercise intolerance. Nor are the 6-min walk test [26], peak myocardial oxygen consumption or brain natriuretic peptide (BNP) obtained routinely, whereas an electrocardiogram and chest X-ray are obtained but are only occasionally helpful for detecting left ventricular hypertrophy or dilatation. Therefore, more sensitive imaging techniques are required for the early detection of myocardial dysfunction. While there is no consensus for the definition of diastolic dysfunction, there have been recent recommendations by the European Society of Cardiology for the diagnosis of diastolic heart failure [27]. These comprise three obligatory conditions: (1) clinical signs and symptoms of congestive heart failure; (2) normal or only mildly abnormal left ventricular systolic function; and (3) evidence of diastolic dysfunction as shown by abnormal left ventricular relaxation, filling, diastolic distensibility or stiffness in addition to elevated levels of BNP. An E/E' ratio [28] (ratio of early diastolic velocities of mitral inflow derived by Doppler imaging and of myocardial movement derived by tissue Doppler imaging) $>15$ is considered diagnostic of diastolic dysfunction and an $\mathrm{E} / \mathrm{E}^{\prime}$ ratio $<8$ as diagnostic of the absence of diastolic heart failure. The use of Dopplerderived mitral inflow measures of left ventricular diastolic function is no longer recommended as a first-line diagnostic approach. An increased left atrial size $\left(>49 \mathrm{ml} / \mathrm{m}^{2}\right)$ and an increased left ventricular mass $\left(>122 \mathrm{~g} / \mathrm{m}^{2}\right.$ in women and 
$>149 \mathrm{~g} / \mathrm{m}^{2}$ in men) are considered sufficient evidence of diastolic dysfunction when the $\mathrm{E} / \mathrm{E}^{\prime}$ ratio is inconclusive.

\section{Pathophysiology of diastolic dysfunction}

Diastole is traditionally divided into four phases: (1) isovolumic relaxation; (2) early diastolic filling, i.e. rapid filling; (3) diastasis; and (4) late diastolic filling, i.e. atrial contraction. Two aspects of diastolic function-relaxation and stiffness-are often considered together, but they describe different properties of the myocardium. Relaxation is a dynamic and energy-consuming myocardial process that begins at the end of contraction and continues throughout isovolumic relaxation and early diastolic filling. Impaired relaxation is either delayed or incomplete and may be due to regional dyssynchrony or to a reduction in energy supply, as in myocardial hypertrophy or ischaemia. Diastolic stiffness is measured at the end of diastole after filling has terminated and is determined by the curvilinear pressure-volume relationship. The slope of the tangent $(\mathrm{d} P / \mathrm{d} v)$ of this relationship defines the chamber stiffness at any given filling pressure. Changes in stiffness may occur as a result of changes in the composition and material properties of the myocardium, such as interstitial fibrosis and left ventricular hypertrophy or both. In the metabolic syndrome and diabetes mellitus, diastolic dysfunction results from abnormal myocardial active relaxation and an increase in passive stiffness due to metabolic derangements and structural remodelling.
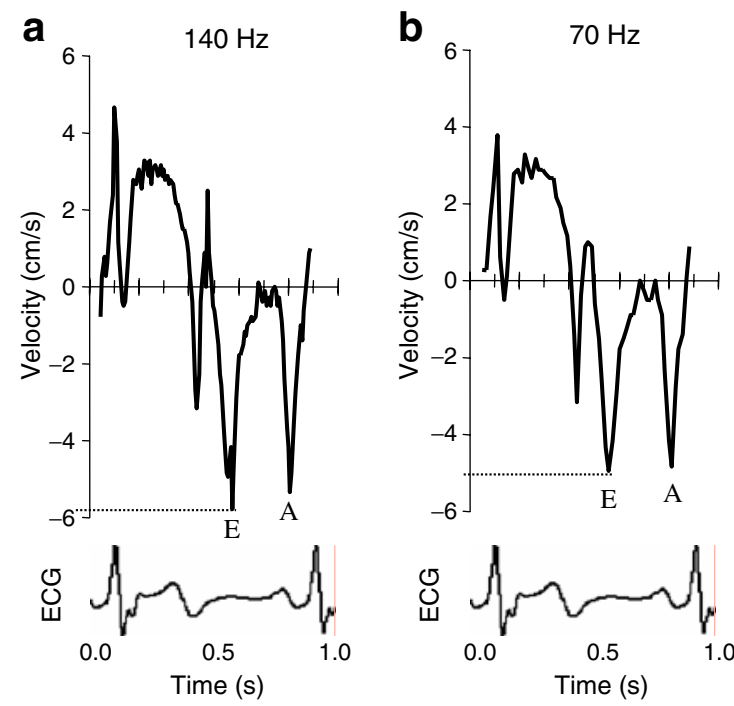

Fig. 1 Dependence of measuring maximal early diastolic velocity $E^{\prime}$ on frame rate. The figure is based on a single-colour M-mode tissue Doppler recording of myocardial velocities. This data set allows calculation of the effects of lowering sampling frequency or frame rates for the display of the resulting $\mathrm{E}$ velocities in the respective
The early diastolic filling period (approximately $100 \mathrm{~ms}$ ) in the normal heart sucks $80 \%$ of the total filling volume into the left ventricle by active energy-consuming myocardial extension. Measurement of maximal early diastolic velocity/function requires high temporal resolution of the imaging technique, i.e. a frame rate $>100$ frame/s, as demonstrated in Fig. 1 [29]. Under-sampling of these rapidly changing velocities leads to underestimation. The optimal time resolution (200/s) is provided in all echocardiographic $\mathrm{M}$-mode recordings and in pulsed tissue Doppler imaging.

Factors involved in the development of diabetic myocardial dysfunction The clustering of cardiovascular risk factors in the metabolic syndrome induces multiple complex metabolic reactions, most prominent among which are altered insulin signalling, glyco- and lipotoxicity, increased cytokine activity and intramyocyte and/or interstitial deposition of triacylglycerol and AGEs, which may all affect myocardial function directly or indirectly [15, 30-34] (Fig. 2). In addition, these multiple risk factors trigger endothelial dysfunction in a summative fashion [35]. Among the effects of endothelial dysfunction are dysregulation of vascular permeability, inflammatory responses and, most prominently, vascular remodelling and atherosclerosis involving the coronary and systemic arteries. This response is mediated by increased vascular tone and associated with increases in arterial stiffness, blood pressure and pulse pressure $[36,37]$. The increase in afterload in the vascular system results in the heart working at an elevated level of resting myocardial oxygen consumption. However, this
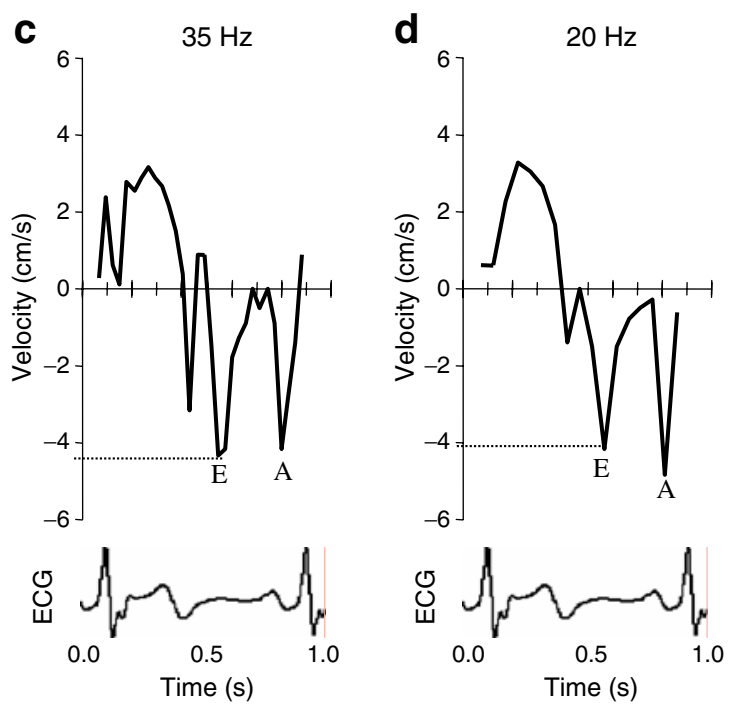

velocity-time plots. Frequency: (a) $140 \mathrm{~Hz}$, (b) $70 \mathrm{~Hz}$, (c) $35 \mathrm{~Hz}$, (d) $20 \mathrm{~Hz}$. If the frame rate is $\leq 35 / \mathrm{s}$, relevant underestimation of early diastolic velocity is induced (J. U. Voigt, University of Leuven, modified from Kukulski et al. [29]) 
Fig. 2 Scheme showing the interrelations between the metabolic syndrome and cardiovascular disease via complex reactions and endothelial dysfunction. These induce vascular stiffness and increased myocardial oxygen consumption in spite of downregulated perfusion. This unfavourable constellation results in cardiomyocyte stress and myocardial diastolic dysfunction as the first stage of diabetic cardiomyopathy

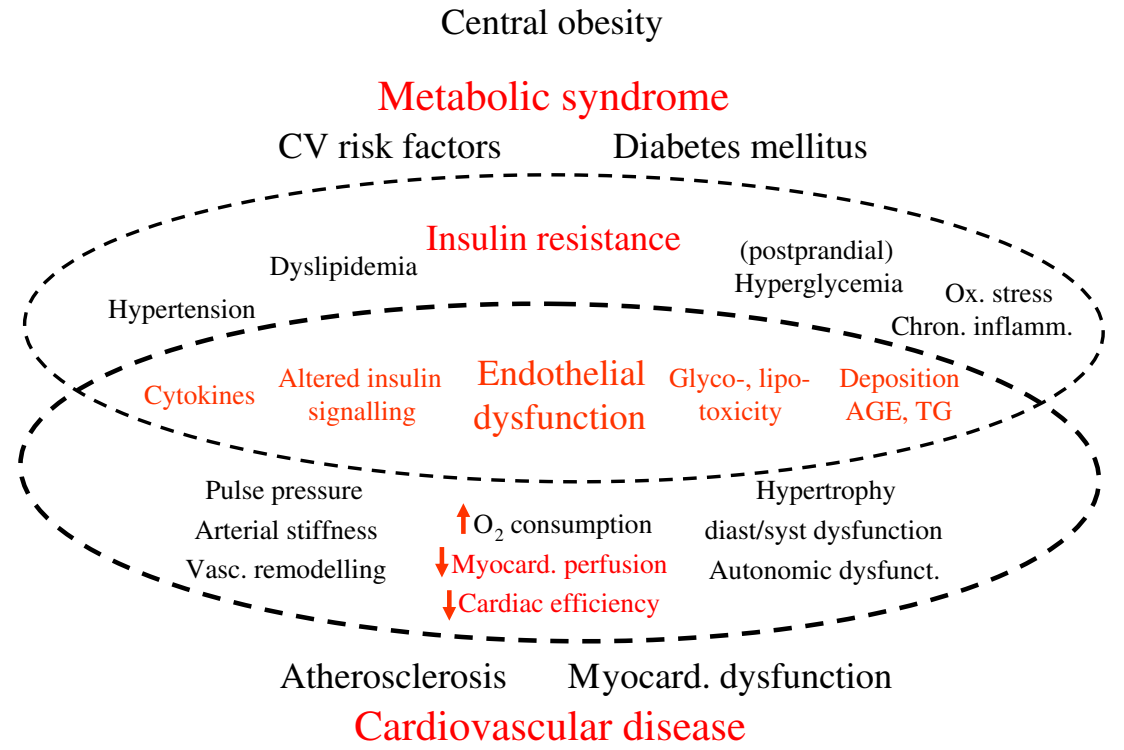

Central obesity

Metabolic syndrome

Atherosclerosis Myocard. dysfunction

Cardiovascular disease

increased energy demand is associated with unfavourable downregulation of myocardial perfusion, impaired intracellular bioenergetics and reduced cardiac efficiency $[15,31$, $38,39]$. This situation may be considered an energy demand/supply mismatch based on metabolically induced abnormalities inducing stress in cardiomyocytes. Subsequently, myocardial hypertrophy, autonomic dysfunction and left ventricular diastolic dysfunction may develop, characterising the first stage of diabetic cardiomyopathy [30]. This stage may remain asymptomatic for a long time and precedes the onset of systolic dysfunction $[6,9,24,40$, 41]. In the late stages of diabetes, diastolic dysfunction may increase further as a result of additional structural abnormalities of the myocardium, such as steatosis cardialis, AGE deposits, interstitial fibrosis and alterations in the extracellular matrix microvasculature that typify the advanced stages of diabetic cardiomyopathy [30, 41-44].

Early diastolic function depends on both myocardial stiffness and energy availability For decades, the pathophysiological mechanisms of impaired left ventricular filling have been considered to be mainly a result of increased myocardial stiffness due to structural changes in the myocardium. Myocardial stiffness affects mainly late diastolic function but may also affect relaxation properties. A recent study based on left ventricular endomyocardial biopsy samples showed that systolic heart failure in diabetic patients was due to increased myocardial stiffness that was mainly associated with AGE deposition and fibrosis. However, the main contributors to diastolic heart failure were high cardiomyocyte resting tension and hypertrophy [41]. Of interest, myocellular hypertrophy in diabetes was unrelated to pressure overload but associated with elevated fasting insulin levels, implicating insulin resistance as a factor contributing to myocyte hypertrophy.

In addition to stiffness, there is also a reversible dynamic component based on the fact that early diastolic relaxation and filling are energy-consuming, active processes. Two well accepted examples of this dynamic component are the immediate and reversible nature of diastolic dysfunction during stress testing [45] and during coronary arterial balloon inflation, which may be regarded as an iatrogenic model of regional and intermittent impairment of myocardial energy supply in man [46]. This model, with an exact onset and offset of perfusion blockade, has confirmed the increased susceptibility of myocardial diastolic function to energy derangements by the occurrence of diastolic dysfunction, not only before but also extending far beyond systolic dysfunction. Limitations in energy supply may arise not only from deficiencies in circulatory transport and perfusion but also from the intracellular processes involved in biochemical energy production and substrate utilisation $[15,31,38,39]$.

Metabolic abnormalities leading to reduced energy availability Abnormal insulin signalling and increased production of reactive oxygen species [32, 33] have recently been demonstrated in individuals with uncomplicated type 2 diabetes [47]. Many of the possible mechanisms of action of reactive oxygen species involve the regulation of energy availability. Indirect effects may involve downregulated perfusion by induction of endothelial dysfunction [35, 48]. Direct effects on cardiomyocytes result in impaired mitochondrial energy production by altered substrate supply and utilisation [38, 49] and mitochondrial uncoupling, which reduces cardiac efficiency [50] and alters insulin signalling. 
The latter plays a key role in insulin resistance [33], reduced bioavailability of nitric oxide [51], altered handling of calcium [52] and mitochondrial damage and dysfunction, culminating in abnormal cardiac remodelling and ventricular dysfunction [36]. Acknowledging diastolic dysfunction as an indicator of reduced myocardial energy availability would provide a better understanding of why augmentation may be observed with short-term improvement of glycaemic control in type 2 diabetes [53] and with acutely increased myocardial perfusion by C-peptide administration in young and athletic individuals with type 1 diabetes [54]. There is an increasing number of reports showing that the extent of hyperglycaemia correlates with diabetic myocardial dysfunction $[30,55,56]$, that good metabolic control reduces the risk of heart failure [57, 58] and that the improvement in diastolic function correlates with the extent of improved glucose control [53, 55, 59]. The optimal glucose-lowering regimen for the protection of patients with diabetes against diastolic heart failure needs to be determined in further studies.

Cardiovascular risk factors determine diastolic function Rapid filling of the left ventricle has been shown to be a sensitive indicator of all types of myocardial damage, being susceptible not only to age and ischaemia but also to virtually all cardiovascular risk factors, including the metabolic syndrome [9, 19, 45, 60-62]. Concordant with these findings is the reversibility of impaired diastolic function, as demonstrated by effective treatment of any of these risk factors, including a sedentary lifestyle and obesity [53, 60, 63-66]. Notably, the reversibility of myocardial abnormalities associated with the metabolic syndrome and diabetes is welcome news for patients and their physicians and is in accord with the recent message from the UK Prospective Diabetes follow-up study, that the most effective treatment is started early in the course of the disease [67].

Age dependence of diastolic function differentiates normal function from dysfunction Myocardial velocity measurements by tissue Doppler imaging clearly demonstrate the predominant influence of ageing on diastolic function [68, 69], confirming previous reports using different imaging techniques. There is a steep and linear decline of normal early diastolic velocity with ageing in normal people, from $16 \mathrm{~cm} / \mathrm{s}$ at the age of 20 years to $6 \mathrm{~cm} / \mathrm{s}$ at 80 years. This decline equals a decrease of $0.16 \mathrm{~cm} / \mathrm{s}$ (i.e. $1 \%$ of the original value) every year. Accordingly, cut-off values for normality vs dysfunction should be defined in a linear regression equation as a function of age [19]. Using pulsed tissue Doppler imaging, the cut-off level for normal agerelated velocity is calculated as $-0.15 \times$ age (years) + $18(\mathrm{~cm} / \mathrm{s})$. This approach allows individual patients to be assigned to normality or dysfunction/risk and avoids the inconsistencies arising from normal values defined for different age groups. There are no sex-related differences in the association of diastolic function and age. $\mathrm{E}^{\prime}$ is also useful in the detection of impaired left ventricular relaxation and estimation of filling pressure $\left(E / E^{\prime}\right)$ in patients with atrial fibrillation [70].

\section{Diagnostics}

Echocardiography and Doppler imaging for traditional left ventricular function assessment The ejection fraction is the traditional measure of systolic left ventricular function and has proved to be a strong predictor of clinical outcome in dilated cardiomyopathy and a variety of cardiac diseases [71, 72]. The ejection fraction bears a curvilinear relationship with end-diastolic volume and an inverse correlation with afterload [72]. However, normalisation by enddiastolic volume makes it insensitive to subclinical myocardial dysfunction in the normal-sized heart or the small hypertrophied ventricle [73]. The normal-sized heart of patients with type 2 diabetes and correspondingly overweight body habitus frequently results in poor delineation of the endocardial borders, which are prerequisites for the quantitative assessment of ejection fraction (Fig. 3, top panels).

Similarly, the traditional measurements of diastolic function, the Doppler-derived mitral valve inflow velocity pattern and its derivatives, have proved difficult to interpret because of the pseudonormal pattern, that defines grade 2 dysfunction, but may be mistaken for the normal pattern [74] (Fig. 3c), unless differentiation by the Valsalva manoeuvre or by assessing pulmonary vein flow is performed. While the clinical course of diastolic dysfunction is characterised by decreasing effectiveness of myocardial relaxation and extension associated with increasing left atrial pressure and size, the respective developments of pressure and filling cannot be mirrored by the traditional Doppler parameters. An even more important limitation, however, is the non-quantitative pattern recognition used for assessing diastolic function and the changes in the course of disease and during preventive therapy.

Pulsed tissue Doppler imaging Detection of systolic or diastolic dysfunction depends on the sensitivity and specificity of the diagnostic technique used. Tissue Doppler imaging has demonstrated that the systolic myocardial velocity $\mathrm{S}^{\prime}$ is a more sensitive measure of systolic function than ejection fraction [75] and that the early diastolic myocardial velocity $\mathrm{E}^{\prime}$ and $\mathrm{E} / \mathrm{E}^{\prime}$ have the best correlation with left ventricular relaxation and compliance indexes 
Systole: LV EF

a
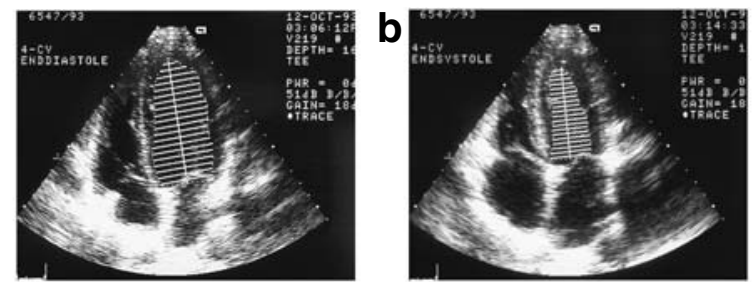

C Diastole: Pattern recognition mitral inflow

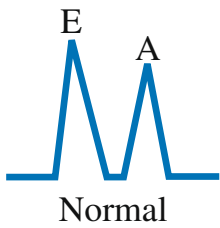

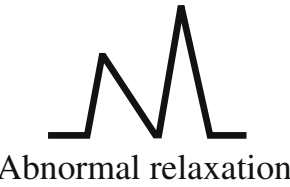

Abnormal relaxation
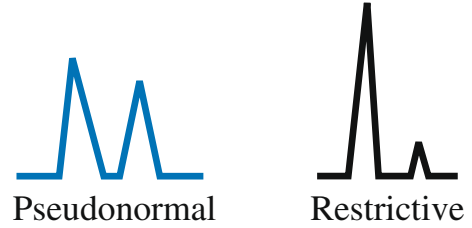

Restrictive
Fig. 3 In the traditional assessment of left ventricular function by twodimensional echocardiography, the systolic variable left ventricular ejection fraction (LV EF) may have poor endocardial border delineation at end-diastole (a) and end-systole (b) as a limiting factor in obese patients. Furthermore, it has limited sensitivity in small-sized hearts.
Traditional variables of diastolic function (c) are the patterns of the early diastolic (E) towards late diastolic (A) Doppler-derived mitral inflow velocities as depicted with increasingly severe dysfunction from left to right. Grade 2 dysfunction, i.e. pseudonormal, may be mistaken for the normal pattern. This pattern recognition is non-quantitative
[76]. Tissue Doppler imaging is a robust and reproducible ultrasound technique [74] employing the low-frequency and high-amplitude ultrasound signals reflected from the myocardium. Regional left ventricular function is quantified as the myocardial velocity of long-axis motion in $\mathrm{cm} / \mathrm{s}$ during systole ( $\left.\mathrm{S}^{\prime}\right)$, early diastole ( $\left.\mathrm{E}^{\prime}\right)$ and late diastole (A'; Fig. 4) [74, 76-78]. Left ventricular global function is the average of these segmental velocities, obtained either at the mitral annulus or very basal myocardium in the apical four- and two-chamber views. The limitations of tissue Doppler imaging include measurement of velocities in apical segments of the left ventricle because of the angle dependency inherent in Doppler techniques. A further problem is the inability to differentiate between actively contracting muscle and passive movements from tethering between myocardial segments. Both limitations are relevant in the evaluation of regional left ventricular function in complicated CAD, which is outside the scope of this review. Tissue Doppler imaging is well validated, sensitive, reproducible, user-friendly and readily available [74]. Furthermore, it is economic, in particular, if pulsed tissue Doppler imaging is being used, which has the additional advantages of optimal temporal resolution, immediate image quality control and online velocity quantification based on fast Fourier transform analysis [45, 74]. Pulsed tissue Doppler imaging enables non-invasive assessment of left ventricular filling pressure by the $\mathrm{E} / \mathrm{E}^{\prime}$ ratio [28] and has been integrated into clinical routine measurements.

Colour Doppler tissue imaging Myocardial velocity measurements can also be obtained using two-dimensional high frame rate (120 frames/min) colour tissue Doppler imaging
Fig. 4 Recordings of velocitytime curves by pulsed tissue Doppler imaging from the basal septum and lateral wall, as indicated in the scheme of the apical four-chamber view measuring systolic $\left(\mathrm{S}^{\prime}\right)$, early diastolic $\left(\mathrm{E}^{\prime}\right)$ and atrial $\left(\mathrm{A}^{\prime}\right)$ myocardial velocity. Global left ventricular function is calculated as the average of these regional segmental velocities. Feasibility is $94 \%$ vs $81 \%$ in two-dimensional echocardiography (modified from von Bibra et al. [45]). LA, left atrium; LV, left ventricle; RA, right atrium; RV, right ventricle

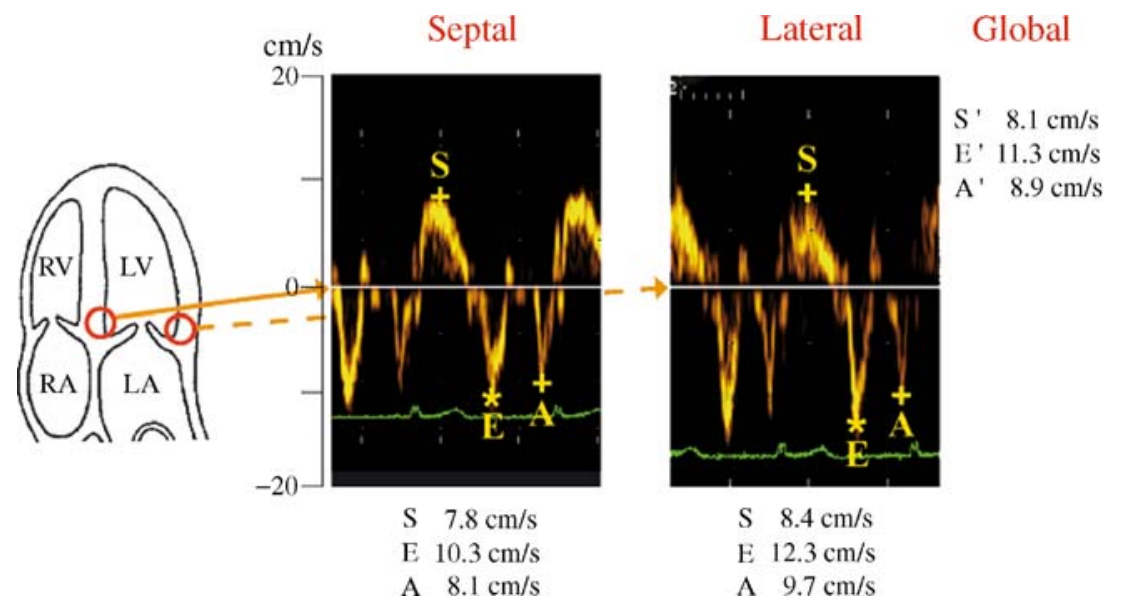


and off-line analysis to reconstruct time--velocity curves. It is important to note that the recording of these velocities is based on autocorrelation and not on fast Fourier analysis as in pulsed tissue Doppler imaging. Accordingly, velocities from colour Doppler imaging are intrinsically lower by $1.8 \pm$ $1.6 \mathrm{~cm} / \mathrm{s}$ compared with velocities derived by pulsed tissue Doppler imaging [29], so that these two techniques should not be interchanged in sequential studies.

Both tissue Doppler techniques have been used successfully to quantify myocardial diastolic and systolic function at rest [9, 19, 28, 30, 60, 75] and during stress [19, 45, 79] and have consistently demonstrated impaired diastolic function in patients with the metabolic syndrome and/or diabetes mellitus type 1 and type 2. Thus, tissue Doppler velocity measurements appear to compensate for the deficiencies of the traditional less precise measures of diastolic function in these patients, and accordingly provide the basis for large-scale therapeutic studies in subclinical and low-grade myocardial disease.

Doppler-based strain and strain rate imaging The theoretical advantage of two-dimensional colour Doppler imaging is that routine images may be acquired for subsequent offline analysis of velocity, strain (change in myocardial length/resting myocardial length) and strain rate (rate of change of strain $[\mathrm{d} S / \mathrm{d} t])$. Strain and strain rate enable assessment of regional and global myocardial mechanical function and appear particularly suitable in CAD and its complications [80] but they have not been integrated into routine clinical practice [74]. However, pulsed tissue Doppler imaging has demonstrated greater sensitivity for the detection of diastolic dysfunction than strain and strain rate $[81,82]$, which is important since diastolic dysfunction commonly precedes systolic dysfunction, especially in CAD, the metabolic syndrome and diabetes mellitus [6, 19, 21, 24, 38, 61, 62].

Speckle tracking-based strain and strain rate imaging The most recent ultrasound development for function assessment, strain and strain rate imaging based on speckle tracking is currently being evaluated [83]. This technology uses ultrasound reflectors within the myocardium to characterise regional myocardium by tracking these reflectors during systolic shortening and relaxation in two and, potentially, three dimensions. Moreover, this technology is not constrained spatially by the angle dependency of Doppler imaging. In addition, speckle tracking allows the evaluation of left ventricular twist and torsion patterns during left ventricular contraction, ejection, relaxation and filling. These patterns are different in systolic heart failure compared with diastolic heart failure and await further clarification [84]. The prognostic value of this technology for the routine assessment of subclinical disease in the metabolic syndrome is not yet known. A limitation of speckle tracking in the assessment of diastolic function is the relatively low frame rate compared with the higher frame rate of pulsed tissue Doppler imaging. Speckle tracking is based on two-dimensional echocardiographic image quality, which may be problematic in overweight patients with the metabolic syndrome and type 2 diabetes. In contrast, pulsed tissue Doppler velocity signals are relatively independent of human tissue attenuation, resulting in velocity recordings that can be analysed even in obese individuals who are difficult to image by twodimensional echocardiography $[45,85]$.

Cine magnetic resonance imaging Cine magnetic resonance imaging (CMR) is the gold standard for the quantitative assessment of left ventricular mass and volumes, from which systolic function may be determined [86]. This non-ionising imaging method has excellent threedimensional spatial resolution. As a consequence of the complex spatial data acquisition in either the gradient echo or the phase-contrast imaging technique, there are some limitations in temporal resolution. Most CMR technologies have a time resolution $\geq 30 \mathrm{~ms}$; that for fast breath-hold gradient echo imaging and CMR fluoroscopy resolution is $15-25 \mathrm{~ms}$ and that for CMR tissue tagging is $\geq 20 \mathrm{~ms}$ [87]. Gradient echo imaging uses repetitive radiofrequency pulses gated to the electrocardiogram so that cine images may be generated during a single breath-hold. Phasecontrast CMR allows velocity encoding of moving structures and blood using the spin phase shift as the basic principle for velocity measurement. Commercially available quantitative CMR software uses spatial modulation of magnetisation (SPAMM) tagging to characterise regional wall motion inhomogeneities [88]. This technique may offer greater accuracy for the detection of regional systolic dysfunction analogous to speckle tracking-derived strain and strain rate. However, it shares with speckle tracking the disadvantages of lower frame rates (50 frames/s) compared with pulsed tissue Doppler imaging, which is not ideal for the assessment of diastolic function. At present, the use of CMR for the assessment of diastolic function may be considered a research tool because of its high cost, limited availability and the need for time-consuming offline data reduction and analysis associated with non-ideal temporal resolution.

The use of contrast agents such as gadolinium to mark scarred myocardium, fibrosis or inflammation by delayed contrast enhancement has been integrated into clinical routine. Magnetic resonance spectroscopy has the potential to recognise changes in myocardial bioenergetics and metabolism, such as increased lipid supply by ${ }^{1} \mathrm{H}$-magnetic resonance spectroscopy and altered high-energy phosphate metabolism assessed by ${ }^{31} \mathrm{P}$-magnetic resonance spectroscopy for measuring the phosphocreatine-ATP ratio $(\mathrm{PCr} /$ 
ATP). These technologies may open new windows on the links between altered metabolism and function in the diabetic heart. First reports demonstrate reduced diastolic function in well controlled patients with uncomplicated type 2 and type 1 diabetes compared with age-matched controls associated with impaired myocardial energy metabolism [38, 49]. Reduced diastolic function has also been observed in type 2 diabetic patients with myocardial steatosis [44] and in middle-aged compared with younger healthy individuals, but is improved in middle-aged individuals on long-lasting aerobic training, paralleled by the PCr/ATP ratio [89].

Summary of imaging techniques Apart from histological and molecular changes in the diabetic myocardium, which are not the focus of this review, there is growing evidence that diastolic and systolic myocardial dysfunction can be and should be routinely quantified by tissue Doppler imaging. Low cost, wide availability, superior temporal resolution and on-line velocity analysis make pulsed tissue Doppler the imaging modality of choice to investigate the presence of left ventricular dysfunction in diabetes or the metabolic syndrome. A specific research advantage of CMR techniques is the ability to assess changes in tissue composition as well as alterations in myocardial metabolism by spectroscopy.

\section{Clinical trials}

Preventive medicine and diastolic function Evidence-based medicine has demonstrated improvement of cardiovascular mortality in individuals with type 2 diabetes mellitus by glucose-lowering drugs and/or other medical therapy, but diastolic function has not been measured. Neither are there any evidence-based studies of patients with diabetes and diastolic heart failure.

Many of the therapeutic principles in cardiovascular endpoint studies have been applied in small studies assessing diastolic function (Table 1). Based on the presumed importance of repetitive hyperglycaemia as the basic mechanism underlying diabetic cardiomyopathy, tests were performed to establish whether improved glycaemic control, preferably with insulin, would reverse left ventricular dysfunction. This was confirmed in two studies of type 2 and one study of type 1 diabetes [53, 55, 59]. In each of these studies, there was a significant inverse correlation between the observed change in diastolic function and the change in glycaemic control. Furthermore, in the first study, there was a parallel improvement in myocardial perfusion [53]. A more recent study [90] of patients with only subtle evidence of diastolic dysfunction [91] and with normal levels of glycaemic control and $\mathrm{E}^{\prime}$ at the beginning of the study demonstrated unchanged diastolic function after further lowering of $\mathrm{HbA}_{1 \mathrm{c}}$. An impact of postprandial glucose control on diastolic function has been suggested in a study comparing the effects of intensified conventional insulin therapy with those of conventional therapy with premixed insulin after 2 years [56] and also in a randomised study comparing analogue with human insulins in intensified conventional insulin regimens [92]. In the insulin analogue treatment arm, improved postmeal glucose values were associated with improved diastolic function, in accord with an earlier study demonstrating improved myocardial perfusion associated with analogue insulins [93]. The alternative to pharmacological glucose-lowering therapy is lifestyle modification, which has also been shown to improve diastolic myocardial function as a result of exercise training $[94,95]$ and restriction of caloric intake [96]. More recently, impressive beneficial effects have been reported on cardiac function and on all components of the metabolic syndrome by a low carbohydrate/high protein diet with a low glycaemic index in spite of a $70 \%$ reduction in glucose-lowering medication, but not by the traditional low fat diet [97, 98].

Glycaemic control is not the only contributor to diastolic dysfunction, as suggested by two detailed studies of diabetic metabolism during treatment with glitazones. An extensive CMR study comparing pioglitazone with metformin [99] showed functional improvement in the glitazone group only but metabolic improvement in both groups, and did not find any correlation between the change in diastolic function and increased myocardial glucose uptake or altered substrate metabolism.

A tissue Doppler imaging crossover study comparing rosiglitazone with glimepiride demonstrated improved fasting and postprandial glucose values and diastolic function for rosiglitazone only. In addition, this study showed a close relationship between the reduction of malondialdehyde (a marker of oxidative stress) and improvement in diastolic function [47].

With regard to cardiovascular preventive medications, first reports describe improved diastolic function and or prognosis with statin therapy [21, 65]. Similarly, improvements in diastolic function have been observed in hypertensive and diabetic patients treated with ACE inhibitors, calcium antagonists, angiotensin II type 1 (AT1) blockers or beta blockers [60, 100-102].

\section{Clinical application of imaging}

Surrogate variable or direct observation of pathological myocardial function In order to screen individuals with the metabolic syndrome or diabetes mellitus for myocardial 


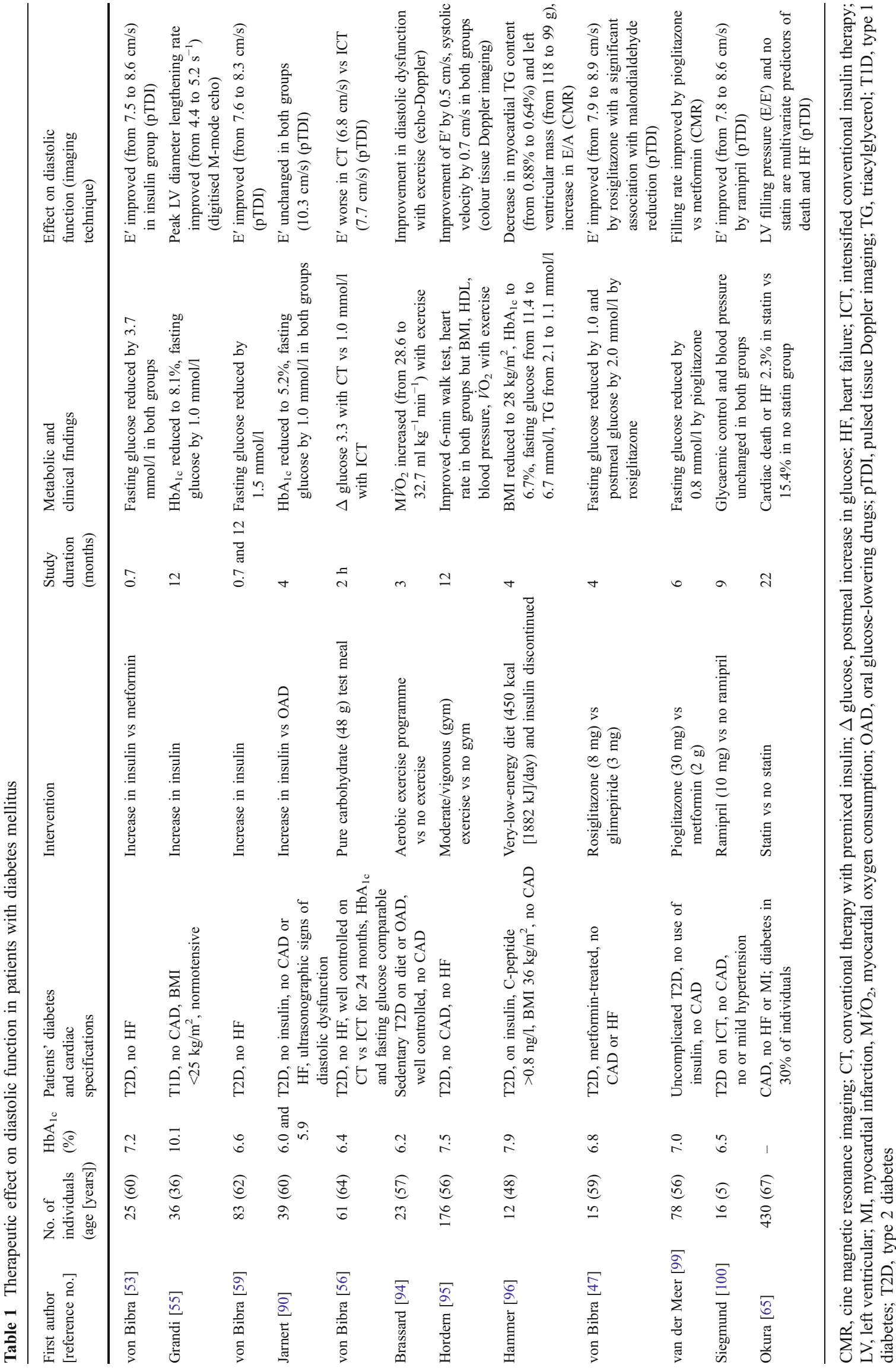


dysfunction, most physicians would prefer a reliable and effective blood test as a surrogate variable compared with the more costly diagnosis by echocardiography. There is increasing evidence that BNP is a sensitive marker of congestive heart failure and acute coronary syndromes [103]. However, BNP does not appear to be a sensitive or specific marker for the early detection of preclinical myocardial dysfunction, in particular diastolic dysfunction $[104,105]$. The sensitivity of BNP testing was reported recently as only $63 \%$ in diabetic patients with subclinical myocardial dysfunction [106]. Furthermore, its potential to monitor therapeutic efficacy appears uncertain, as suggested by the outcome of the Steno-2 Study in 160 patients with type 2 diabetes mellitus and microalbuminuria. In this study, multimodal therapy induced significant improvement in metabolic control and cardiovascular risk factors, but BNP increased significantly [107]. Thus, the use of BNP cannot be considered effective for the assessment of subclinical diastolic dysfunction.

Preventive cardiovascular medicine should ideally use diagnostic measurements that involve direct observation of the pathological changes in the myocardium rather than rely upon identification of epiphenomenal risk factors or surrogate biomarkers. At present, however, it is premature to make recommendations about when, how often and which selection of diabetic patients should be imaged to assess baseline diastolic function. The clinical picture, in particular a reduced exercise capacity limiting normal daily activities, should raise suspicion of undiagnosed diastolic dysfunction and initiate referral to a cardiologist for diagnostic ultrasonography. Similarly, a combination of obesity, insulin resistance, mediocre glycaemic control and further cardiovascular risk factors or CAD should indicate a diagnostic assessment for early preventive action. Because of the current lack of evidence-based therapeutic strategies, preventive action should focus on addressing the underlying pathophysiological mechanisms of the metabolic disease by at least recommending lifestyle changes. Regular physical training/activity and dietary modification lack side effects and have had a success rate better than that of preventive drug therapy, at least in CAD patients [108].

Highly sensitive variables are needed for individual patient management Whereas the clinical endpoints that are used in evidence-based preventive medicine are often unsuitable for tailoring individual patient therapy, the quantification of ideal sensitive and routinely available current variables is required in order to demonstrate therapeutic efficacy. Many of the large clinical trials report the significance of small numerical differences between the control group and the active treatment arms, but this has little or no clinical relevance for individual patients. Accordingly, it remains of clinical interest to study highly sensitive variables, which have the potential to become significant in small patient numbers, e.g. in eight to 15 observed cases. These variables may yield meaningful individual differences when compared before and after therapeutic interventions, as has been shown to be true for diastolic myocardial velocity quantified by pulsed tissue Doppler imaging and reported to be suitable for supporting therapeutic decision-making $[47,53,54,100]$.

\section{Conclusion}

The cardiovascular disease associated with the metabolic syndrome and with diabetes mellitus comprises vascular and myocardial abnormalities. This myocardial dysfunction is characterised predominantly by diastolic dysfunction consisting of relaxation abnormalities that are prevalent and have prognostic importance. Diastolic dysfunction can be detected and quantified by pulsed tissue Doppler imaging as a clinical routine and assigned to normality vs dysfunction by using the linear inverse relation of individual age to diastolic function. Diastolic dysfunction is a direct measure of structural and dynamic, metabolically induced, pathophysiological processes in the myocardium. Tissue Doppler imaging has the potential to meet the present need for a robust tool that can establish therapeutic efficacy in future preventive studies of insulin resistance, diabetes and cardiovascular disease and, thus, should be used to identify (pre-) diabetic individuals with diastolic dysfunction and to optimise their clinical management.

Acknowledgements This work was supported in part by grants to H. von Bibra by the Heidenhain Foundation, the Swedish Technical Research Council, the Swedish Heart Lung Foundation, the Swedish Medical Research Council (project no. 11201), the German Cardiac Society and Baden-Wuertemberg LVA Pension System. M. St John Sutton is funded by the Commonwealth of Pennsylvania.

Duality of interest The authors declare that there is no duality of interest associated with this manuscript.

Open Access This article is distributed under the terms of the Creative Commons Attribution Noncommercial License which permits any noncommercial use, distribution, and reproduction in any medium, provided the original author(s) and source are credited.

\section{References}

1. Hanefeld M, Leonhardt W (1981) Das metabolische Syndrom. Dtsch Gesundheitsw 36:545-551, article in German

2. Ford ES, Giles WH, Dietz WH (2002) Prevalence of the metabolic syndrome among US adults: findings from the third national health and nutrition examination survey. JAMA 287:356-359

3. Reavan GM (1988) Banting lecture 1988. Role of insulin resistance in human disease. Diabetes 37:1595-1607

4. Diamant M, Tushuisen ME (2006) The metabolic syndrome and endothelial dysfunction: common highway to type 2 diabetes and CVD? Curr Diab Rep 6:279-286 
5. Norhammar A, Tenerz A, Nilsson G, Hamsten A, Efendic S, Rydén L (2003) Glucose metabolism in patients with acute myocardial infarction and no previous diagnosis of diabetes mellitus: a prospective study. Lancet 359:2140-2144

6. Celentano A, Vaccaro O, Tammaro P et al (1995) Early abnormalities of cardiac function in non-insulin-dependent diabetes mellitus and impaired glucose tolerance. Am J Cardiol 76:1173-1176

7. Raev DC (1994) Which left ventricular function is impaired earlier in the evolution of diabetic cardiomyopathy? An echocardiographic study of young type I diabetic patients. Diabetes Care 17:633-639

8. Rubler S, Dlugash J, Yuceoglu YZ, Kumral T, BranWood AW, Grishman A (1972) New type of cardiomyopathy associated with diabetic glomerulosclerosis. Am J Cardiol 30:595-602

9. De las Fuentes L, Brown AL, Mathews SJ et al (2007) Metabolic syndrome is associated with abnormal left ventricular diastolic function independent of left ventricular mass. Eur Heart J 28:553559

10. Bella JN, Palmieri V, Roman MJ et al (2002) Mitral ratio of peak early to late diastolic filling velocity as a predictor of mortality in middle-aged and elderly adults. The Strong Heart Study. Circulation 105:1928-1933

11. Wang M, Yip G, Wanf A et al (2003) Peak early diastolic mitral annulus velocity by tissue Doppler imaging adds independent and incremental prognostic value. J Am Coll Cardiol 43:820-826

12. Kitzman DW, Gardin JM, Gottdiener JS et al (2001) Importance of heart failure with preserved systolic function in patients $\geq 65$ years of age. Am J Cardiol 87:413-419

13. Owan TE, Hodge DO, Herges RM, Jacobson SJ, Roger VL, Redfield MM (2006) Trends in prevalence and outcome of heart failure with preserved ejection fraction. N Engl J Med 355:251-259

14. Bartnik M, Malmberg K, Rydén L (2003) Diabetes and the heart: compromised myocardial function - a common challenge. Eur Heart J Suppl 5(Suppl B):B33-B41

15. Boudina S, Dale Abel E (2007) Diabetic cardiomyopathy revisited. Circulation 115:3213-3223

16. Malmberg K, Rydén L, Efendic S et al (1995) A randomized trial of insulin-glucose infusion followed by subcutaneous insulin treatment in diabetic patients with acute myocardial infarction (DIGAMY Study): effects on mortality at 1 year. J Am Coll Cardiol 26:57-65

17. de Groote P, Herpin D, Diévart F et al (2008) Treatment of heart failure with preserved systolic function. Arch Cardiovasc Dis 101:361-372

18. Fischer M, Baessler A, Hense HW et al (2003) Prevalence of left ventricular diastolic dysfunction in the community. Results from a Doppler echocardiographic-based survey of a population sample. Eur Heart J 24:320-328

19. von Bibra H, Thrainsdottir IS, Hansen A et al (2005) Tissue Doppler imaging for the detection and quantitation of myocardial dysfunction in patients with type 2 diabetes mellitus: a methodological study. Diabetes Vasc Dis Res 2:483-487

20. Bell DS (2003) Diabetic cardiomyopathy. Diabetes Care 26:2949-2951

21. Dounis V, Siegmund T, Jensen J et al (2006) Global myocardial perfusion and diastolic function are impaired to a similar extent in patients with type 2 diabetes mellitus and in patients with coronary artery disease - evaluation by contrast echocardiography and pulsed tissue Doppler. Diabetologia 49:2729-2740

22. Poirier P, Garneau C, Bogaty P et al (2000) Impact of left ventricular diastolic dysfunction on maximal treadmill performance in normotensive subjects with well-controlled type 2 diabetes mellitus. Am J Cardiol 85:473-477

23. Levinger I, Goodman C, Hare DL, Jerums G, Selig S (2007) The effect of resistance training on functional capacity and quality of life in individuals with high and low numbers of metabolic risk factors. Diabetes Care 30:2205-2210

24. O'Rourke MF (2001) Diastolic heart failure, diastolic left ventricular dysfunction and exercise intolerance. J Am Coll Cardiol 38:803-805

25. Saraiva RM, Duarte DM, Duarte MP et al (2005) Tissue Doppler imaging identifies asymptomatic normotensive diabetics with diastolic dysfunction and reduced exercise tolerance. Echocardiography 22:561-570

26. Bittner V, Weiner DH, Yusuf S et al (1993) Prediction of mortality and morbidity with a 6-minute walk test in patients with LV dysfunction. SOLVD Investigators JAMA 270:17021707

27. Paulus WJ, Tschöpe C, Rusconi C et al (2007) How to diagnose diastolic heart failure: a consensus statement on the diagnosis of heart failure with normal left ventricular ejection fraction by the Heart Failure and Echocardiography Associations of the European Society of Cardiology. Eur Heart J 28:2539-2550

28. Nagueh SF, Middleton KJ, Kopelen HA et al (1997) Doppler tissue imaging: a noninvasive technique for evaluation of left ventricular relaxation and estimation of filling pressures. J Am Coll Cardiol 30:1527-1533

29. Kukulski T, Voigt JU, Wilkenshoff UM et al (2000) Comparison of regional myocardial velocity information derived by pulsed and colour Doppler techniques. An in vitro and in vivo study. Echocardiography 17:639-651

30. Fang ZY (2004) Diabetic cardiomyopathy: evidence, mechanisms and therapeutic implications. Endocr Rev 25:543-567

31. Brownlee M (2005) The pathophysiology of diabetic complications - a unifying mechanism. Diabetes 54:1615-1625

32. Ouwens DM, Diamant M (2007) Myocardial insulin action and the contribution of insulin resistance to the pathogenesis of diabetic cardiomyopathy. Arch Physiol Biochem 113:76-85

33. Houstis N, Rosen ED, Lander ES (2006) Reactive oxygen species have a causal role in multiple forms of insulin resistance. Nature 440:944-948

34. Young ME, McNulty P, Taegtmeyer H (2002) Adaptation and maladaptation of the heart in diabetes. II. Potential mechanisms. Circulation 105:1861-1870

35. Widlansky ME, Gokce N, Keaney JF et al (2003) The clinical implications of endothelial dysfunction. J Am Coll Cardiol 42:1149 1160

36. Wilkinson IB, Franklin SS, Cockroft JR (2004) Nitric oxide and the regulation of large artery stiffness: from physiology to pharmacology. Hypertension 44:112-116

37. Scuteri A, Najjar SS, Muller DC, Andres R et al (2004) Metabolic syndrome amplifies the age-associated increases in vascular thickness and stiffness. J Am Coll Cardiol 43:1388-1395

38. Diamant M, Lamb HJ, Groeneveld Y et al (2003) Diastolic dysfunction is associated with altered myocardial metabolism in asymptomatic normotensive patients with well-controlled type 2 diabetes mellitus. J Am Coll Cardiol 42:328-335

39. Nitenberg A, Valensi P, Sachs R et al (1993) Impairment of coronary vascular reserve and $\mathrm{ACh}$ induced coronary vasodilatation in diabetic patients with angiographically normal coronary arteries and normal left ventricular systolic function. Diabetes 42:1017-1025

40. Karamitsos TD, Karvounis HI, Didangelos T, Parcharidis GE, Karamitsos DT (2008) Impact of autonomic neuropathy on left ventricular function in normotensive type 1 diabetic patients: a tissue Doppler echocardiographic study. Diabetes Care 31:325-327

41. van Heerebeek L, Hamdani N, Handoko L et al (2008) Diastolic stiffness of the failing heart: importance of fibrosis, advanced glycation endproducts and myocyte resting tension. Circulation 117:43-51 
42. Bierhaus A, Schiekofer S, Schwaninger M et al (2001) Diabetes associated sustained activation of the transcription factor nuclear factor-kappa $\beta$. Diabetes 50:2792-2808

43. Westermann D, Kasner M, Stendijk P et al (2008) Role of left ventricular stiffness in heart failure with normal ejection fraction. Circulation 117:2051-2060

44. Rijzewjik LJ, van der Meer RW, Smit JW et al (2008) Myocardial steatosis is an independent predictor of diastolic dysfunction in type 2 diabetes mellitus. J Am Coll Cardiol 52:1793-1799

45. von Bibra H, Tuchnitz A, Klein A et al (2000) Regional diastolic function by pulsed Doppler myocardial mapping for the detection of left ventricular ischaemia during pharmacologic stress testing - a comparison with stress echocardiography and perfusion scintigraphy. J Am Coll Cardiol 36:444-452

46. Wijns W, Serruys PW, Slager C et al (1986) Effect of coronary occlusion during percutaneous transluminal angioplasty in humans on left ventricular chamber stiffness and regional diastolic pressure-radius relations. J Am Coll Cardiol 7:455-463

47. von Bibra H, Diamant M, Scheffer PG et al (2008) Rosiglitazone, but not glimepiride, improves myocardial diastolic dysfunction in association with a reduction of oxidative stress in type 2 diabetes patients without overt heart disease. Diabetes Vasc Dis Res 5:310 318

48. Scognamiglio R, Negut C, de Kreutzenberg S et al (2005) Postprandial myocardial perfusion in healthy subjects and in type 2 diabetic patients. Circulation 112:179-184

49. Metzler B, Schocke MF, Steinboek P et al (2002) Decreased high energy phosphate ratios in the myocardium of men with diabetes mellitus type 1. J Cardiovasc Magn Reson 4:493-502

50. Boudina S, Abel ED (2006) Mitochondrial uncoupling: a key contributor to reduced cardiac efficiency in diabetes. Physiology 21:250-258

51. Wold LE, Dutta K, Mason MM et al (2005) Impaired SERCA function contributes to cardiomyocyte dysfunction in insulin resistant rats. J Mol Cell Cardiol 39:297-307

52. Barouch LA, Harrison RW, Skaf MW et al (2002) Nitric oxide regulates the heart by spatial confinement of nitric oxide synthase isoforms. Nature 426:337-339

53. von Bibra H, Hansen A, Dounis V et al (2004) Insulin based improved metabolic control augments myocardial diastolic function and perfusion in patients with type 2 diabetes mellitus. Heart 90:1483-1484

54. Hansen A, Johansson BL, Wahren J, von Bibra H (2002) Beneficial effects of C-peptide on myocardial function and perfusion in patients with type 1 diabetes. Diabetes 51:3077-3082

55. Grandi AM, Piantanida E, Franzetti I et al (2006) Effect of glycemic control on left ventricular diastolic function in type 1 diabetes mellitus. Am J Cardiol 97:71-76

56. von Bibra H, Siegmund T, Ceriello A et al (2009) Optimized postprandial glucose control is associated with improved cardiac/ vascular function - comparison of three insulin regimens in well controlled patients with type 2 diabetes. Hormone Metab Res 41:109-115

57. Iribarren C, Karter AJ, Go AS et al (2001) Glycemic control and heart failure among adult patients with diabetes. Circulation 103:2668-2673

58. Held C, Gerstein HC, Yusuf S, for the ONTARGET/TRANSCEND Investigators et al (2007) Glucose levels predict hospitalization for congestive heart failure in patients at high cardiovascular risk. Circulation 115:1371-1375

59. von Bibra H, Siegmund T, Hansen A, Jensen J, SchummDraeger PM (2007) Improved myocardial function by improved metabolic control in patients with type 2 diabetes mellitus. Dtsch Med Wochenschr 132:729-734
60. Di Bello V, Giorgi D, Pedrinielli R et al (2004) Left ventricular hypertrophy and its regression in essential arterial hypertension. A tissue Doppler imaging study. Am J Hypertens 17:882-890

61. Grandi AM, Zanzi P, Piantanida E et al (2000) Obesity and left ventricular diastolic function: noninvasive study in normotensives and newly diagnosed never-treated hypertensives. Int $\mathrm{J}$ Obes 24:954-958

62. Peterson LR, Waggoner AD, Schlechtman KB et al (2004) Alterations in left ventricular structure and function in young healthy obese women: assessment by echocardiography and tissue Doppler imaging. J Am Coll Cardiol 43:1399-1404

63. Forman DE, Manning DJ, Hauser R et al (1992) Enhanced LV diastolic filling associated with long term endurance training. $\mathrm{J}$ Gerontol 47:M56-M58

64. Hoffmann U, Globits S, Stefenelli T et al (2001) The effects of ACE inhibitor therapy on LV myocardial mass and diastolic filling in previously untreated hypertensive patients: a cine MRI study. J Magn Reson Imaging 14:16-22

65. Okura H, Asawa K, Kubo T et al (2007) Impact of statin therapy on systemic inflammation, left ventricular systolic and diastolic function and prognosis in low risk ischemic heart patients without history of heart failure. Intern Med 47:1337-1343

66. Wong CY, Byrne NM, O’Moore-Sullivan T et al (2006) Effect of weight loss due to lifestyle intervention on subclinical cardiovascular dysfunction in obesity (body mass index $>30 \mathrm{~kg} / \mathrm{m}^{2}$ ). Am J Cardiol 98:1593-1598

67. Holman RR, Paul SK, Bethel MA, Matthews DR, Neil HA (2008) 10-years follow-up of intensive glucose control in type 2 diabetes. N Engl J Med 359:1577-1589

68. Inelli P, Sanchez R, Marra F et al (2008) The impact of aging on left ventricular longitudinal function in healthy subjects: a pulsed tissue Doppler study. Eur J Echocardiog 9:241-249

69. Nikitin N, Witte K, Thackray S et al (2003) Longitudinal ventricular function: normal values of atrioventricular annular and myocardial velocities measured with quantitative two-dimensional color Doppler tissue imaging. J Am Soc Echocardiog 16:906-921

70. Okura N, Takada Y, Kubo T et al (2006) Tissue Dopplerderived index of left ventricular filling pressure, $E / E^{\prime}$, predicts survival of patients with non-valvular atrial fibrillation. Heart 92:1248-1252

71. Applegate RJ, Little WC (1991) Systolic and diastolic left ventricular function. Prog Cardiol 4:63-72

72. Gaasch WH, Delorey DE, St John Sutton MG et al (2008) Patterns of structural and functional remodeling of the left ventricle in chronic heart failure. Am J Cardiol 102:459-462

73. Aurigemma GP, Silver KH, Priest MA et al (1995) Geometric changes allow normal ejection fraction despite depressed myocardial shortening in hypertensive left ventricular hypertrophy. J Am Coll Cardiol 26:195-202

74. Yu CM, Sanderson JE, Marwick TH et al (2007) Tissue Doppler imaging. A new prognosticator for cardiovascular disease. J Am Coll Cardiol 49:1903-1914

75. Yip G, Wang M, Zhang Y et al (2002) Left ventricular long axis function in diastolic heart failure is reduced in both diastole and systole: time for a redefinition? Heart 87:121-125

76. Kasner M, Westermann D, Steendijk P et al (2007) Utility of Doppler echocardiography and tissue Doppler imaging in the estimation of diastolic function in heart failure with normal ejection fraction - a comparative Doppler-conductance catheterization study. Circulation 116:637-647

77. Gorcsan J, Strum DP, Mandarino WA et al (1997) Quantitative assessment of alterations in regional left ventricular contractility with color-coded tissue Doppler echocardiography. Comparison with sonomicrometry and pressure volume relations. Circulation 95:2423-2433 
78. Nagueh SF, Sun H, Kopelen HA et al (2001) Hemodynamic determinants of the mitral annulus diastolic velocities by tissue Doppler. J Am Coll Cardiol 37:278-285

79. Cain P, Khoury V, Short L, Marwick TH (2003) Usefulness of quantitative echocardiographic techniques to predict recovery of regional and global left ventricular function after acute myocardial infarction. Am J Cardiol 91:391-396

80. Marwick T (2006) Measurement of strain and strain rate by echocardiography: ready for prime time? J Am Coll Cardiol 47:1313-1327

81. Fang ZY, Yuda S, Anderson V, Short L, Case C, Marwick TH (2003) Echocardiographic detection of early diabetic myocardial disease. J Am Coll Cardiol 41:611-617

82. Koyama J, Ray-Sequin PA, Falk RH (2003) Longitudinal myocardial function assessed by tissue velocity, strain and strain rate tissue Doppler echocardiography in patients with $\mathrm{AL}$ (primary) cardiac amyloidosis. Circulation 107:2446-2452

83. Helle-Valle T, Crosby J, Edvardsen T et al (2005) New noninvasive method for assessment of left ventricular rotation: speckle tracking echocardiography. Circulation 112:3149-3156

84. Gillebert TC, van de Veire NR (2008) About left ventricular torsion, sex differences, shear strain, and diastolic heart failure. Eur Heart J 29:1215-1217

85. Bess RL, Khan S, Rosman HS, Cohen GI, Alleban Z, Gardin JM (2006) Technical aspects of diastology: why mitral inflow and tissue Doppler imaging are the preferred parameters? Echocardiography 23:332-339

86. Pennel DJ, Sechtem UP, Higgins B et al (2004) Working Group on Cardiovascular Magnetic Resonance of the European Society of Cardiology. Clinical indications for cardiovascular magnetic resonance (CMR): Consensus Panel report. Eur Heart J 25:1940-1965

87. Paelinck BP, Lamb H, de Bax JJ, van der Wall EE, Roos A (2002) Assessment of diastolic function by cardiovascular magnetic resonance. Am Heart J 144:198-205

88. Götte MU, Germans T, Russell IK et al (2006) Myocardial strain and torsion quantified by cardiovascular magnetic resonance tissue tagging studies in normal and impaired left ventricular function. J Am Coll Cardiol 48:2002-2011

89. Perseghin G, de Cobelli F, Esposito A et al (2009) Left ventricular function and energy metabolism in middle-aged men undergoing long-lasting sustained aerobic oxidative training. Heart 95:630-635

90. Jarnert C, Landstedt-Hallin L, Malmberg K et al (2009) A randomized trial of the impact of strict glycaemic control on myocardial function and perfusion reserve: a report from the DADD (Diabetes Mellitus And Diastolic Dysfunction) Study. Eur J Heart Fail 11:39-47

91. van Heerebeek L, Paulus WJ (2009) The dialogue between diabetes and diastole. Eur J Heart Fail 11:3-5

92. von Bibra H, Riemer M, Walther I et al (2009) Cardiac function and post-meal glucose are improved by 24 months of analogue vs human insulin therapy in patients with type 2 diabetes with intensive conventional insulin therapy (ICT). Diabetes 58(Suppl 1):A123

93. Scognamiglio R, Negut C, de Kreutzenberg S, Tiengo A, Avogaro A (2006) Effects of different insulin regimens on postprandial myocardial perfusion defects in type 2 diabetic patients. Diabetes Care 29:95-100

94. Brassard P, Legault S, Garneau C, Bogaty P, Dumesnil JG, Poirier P (2007) Normalization of diastolic dysfunction in type 2 diabetics after exercise training. Med Sci Sports Exerc 39:18961901

95. Hordern MD, Coombes JS, Cooney LM, Jeffriess L, Prins JB, Marwick TH (2009) Effects of exercise intervention on myocardial function in type 2 diabetes. Heart 95:1343-1349

96. Hammer S, Snel M, Lamb HJ et al (2008) Prolonged caloric restriction in obese patients with type 2 diabetes mellitus decreases myocardial triglyceride content and improves myocardial function. J Am Coll Cardiol 52:1006-1012

97. Westman EC, Feinman RD, Mavropoulos JC et al (2007) Lowcarbohydrate nutrition and metabolism. Am J Clin Nutr 86:276284

98. von Bibra H, Wulf G, Heilmeyer P (2009) A Low Glycemic and Insulinemic Diet (LOGI ${ }^{\circledR}$ ) and moderate physical training improve cardiac function and metabolic syndrome in spite of a $70 \%$ reduction of antidiabetic therapy. Diabetes 58 (Suppl 1): A95

99. van der Meer RW, Rijzewijk LJ, de Jong HW et al (2009) Pioglitazone improves cardiac function and alters myocardial substrate metabolism without affecting cardiac triglyceride accumulation and high-energy phosphate metabolism in patients with well-controlled type 2 diabetes mellitus. Circulation 119:2069-2077

100. Siegmund T, Schumm-Draeger PM, Antoni D, von Bibra H (2007) Beneficial effects of ramipril on myocardial diastolic function in patients with type 2 diabetes mellitus, normal LV function and without coronary artery disease - a prospective study using tissue Doppler. Diabetes Vasc Dis Res 4:358-364

101. Müller-Brunotte R, Kahan T, Malmqvist K et al (2006) Tissue velocity echocardiography shows early improvement in diastolic function with irbesartan and atenolol therapy in patients with hypertensive left ventricular hypertrophy. Results from the Swedish Irbesartan Left Ventricular Hypertrophy Investigation vs Atenolol (SILVHIA). Am J Hypertens 19:927-936

102. Onose Y, Oki T, Yamada H et al (2001) Effect of cilnidipine on left ventricular diastolic function in hypertensive patients as assessed by pulsed Doppler echocardiography and by pulsed tissue Doppler imaging. Jpn Circ J 65:305-309

103. Pfister R, Schneider CA (2004) Natriuretic peptides BNP and NT-pro-BNP: just established laboratory markers in clinical practice or just perspectives? Clin Chim Acta 349:25-38

104. Vasan RS, Benjamin EJ, Larson MG et al (2002) Plasma natriuretic peptides for community screening for left ventricular hypertrophy and systolic dysfunction. The Framingham Heart Study. JAMA 288:1252-1259

105. Redfield MM, Rodeheffer RJ, Jacobsen SJ et al (2004) Plasma brain natriuretic peptide to detect preclinical ventricular systolic or diastolic dysfunction: a community-based study. Circulation 109:376-381

106. Fang ZY, Schull-Meade R, Leano R et al (2005) Screening for heart disease in diabetic subjects. Am Heart J 149:349-354

107. Gaede P, Hildebrandt P, Hess G et al (2005) Plasma N-terminal pro-brain natriuretic peptide as a major risk marker for cardiovascular disease in patients with type 2 diabetes and microalbuminuria. Diabetologia 48:156-163

108. Iestra JA, Kromhout D, van der Schouw YT, Grobbee DE, Boshuizen HC, van Staveren WA (2005) Effect size estimates of lifestyle and dietary changes on all-cause mortality in coronary artery disease patients. Circulation 112:924-934 